\title{
Helping Individuals to Understand Synergistic Risks: An Assessment of Message Contents Depicting Mechanistic and Probabilistic Concepts
}

\author{
RUNNING HEADER:
}

Communicating Synergsitic Risk

\section{AUTHOR NAMES AND AFFILIATIONS:}

Ian G.J. Dawson*, Centre for Risk Research, School of Management, Faculty of Business and Law, University of Southampton, UK.

Johnnie E.V. Johnson, Centre for Risk Research, School of Management, Faculty of Business and Law, University of Southampton, UK.

Michelle A. Luke, Centre for Risk Research, School of Management, Faculty of Business and Law, University of Southampton, UK.

*Address correspondence to Ian G.J. Dawson, Centre for Risk Research, School of Management, Faculty of Business and Law, University of Southampton, SO17 1BJ, UK.

\section{ACKNOWLEDGMENTS:}

The authors would like to thank Paul Goodwin, Sally Brailsford, Simone Dohle and two anonymous reviewers for their helpful and insightful comments regarding an earlier draft of this paper. 


\section{ABSTRACT}

Accumulating evidence shows that certain hazard combinations interact to present synergistic risks. However, little is known about the most effective ways of helping individuals to understand this complex risk concept. More specifically, there is an absence of empirical research that has assessed the relative efficacy of messages that explain either the causal mechanism and/or the probabilistic components of synergistic risks. In an experiment designed to address this issue, we presented participants with messages concerning the synergistic risk of developing esophageal cancer for individuals who consume both tobacco and alcohol. Relative to a control group, we compared the extent to which messages featuring content detailing the underlying biological mechanism, the probabilistic risk, or both, improved understanding of the synergistic risk. Our results showed that messages containing details of both the mechanism and probabilistic information were most effective at enabling individuals to understand that the alcohol-tobacco combination presents a synergistic risk. In addition, large improvements in the accuracy of cancer frequency estimates were observed amongst individuals who received probabilistic information, and the highest relative increase in professed willingness to adopt precautionary behaviors was observed amongst individuals who received the mechanism information only. Importantly, these findings could be utilized in the development of a general model for the communication of synergistic risks. Furthermore, in contrast to previous findings, our study demonstrates that risk messages can be both effective and efficient in helping individuals to acquire a greater understanding of synergistic risks. Acquiring such knowledge could lead to significant improvements in riskrelated decisions concerning combined hazards.

KEYWORDS: Biological mechanism; Numeracy; Probability; Risk communication; Synergistic risk 


\section{INTRODUCTION}

Scientific evidence shows that the risk attributable to certain hazard combinations exceeds the sum of the risk attributable to each constituent hazard (i.e., the combination presents a 'synergistic risk'). For example, epidemiological studies have found that the combined use of alcohol and tobacco results in a synergistic risk of laryngeal and esophageal cancer, and interactions between particular drugs, such as aspirin and warfarin, result in a synergistic risk of serious internal bleeding. ${ }^{(1-3)}$ Further examples include a synergistic increase in the incidence of psychosis amongst individuals living in urban areas and who have a family history of mental illness, and a synergistic risk of species extinction that is attributable to the interaction of climate change and habitat destruction. ${ }^{(4,5)}$ Evidence of synergistic risks, particularly in the health domain, continues to accumulate. ${ }^{(6,7)}$

Several studies investigating subjective risk judgments for combined hazards have indicated that individuals often judge synergistic risks as either additive (i.e., equal to the sum of the risk attributable to each constituent hazard) or sub-additive (i.e., less than the sum of the risk attributable to each constituent hazard) risks (for detailed reviews see references ${ }^{8,9}$ ). These findings raise the concern that such underestimations of risk may impede an individual's motivation to adopt protective behaviors or to demand, political and economic actions to address synergistic risks. ${ }^{(9,10)}$ A further concern is that the failure to understand particular synergistic risks by policy-makers, regulators, and medical professionals may inhibit the implementation of relevant precautionary interventions. ${ }^{(11,12)}$ These concerns highlight the value of identifying effective and efficient methods of helping individuals to understand specific synergistic risks. However, empirical research regarding the communication of the nature of synergistic risks is scarce and the communication approaches that have been assessed have either been found to be unsuccessful or dependent on complex and time-consuming procedures. ${ }^{(9,13,14)}$ Consequently, these approaches could not be 
employed to efficiently bring specific synergistic risks to the attention of large groups, such as regulators, policy-makers, health practitioners, and the wider public.

Hence, there are unanswered questions concerning the most effective methods for conveying information about synergistic risks in a manner that is both efficient and easily understood by the recipients. ${ }^{(15)}$ Our study helps to fill some of these gaps in our knowledge and, consequently, provides important insights that could be employed in the development of a general model concerning the effective and efficient communication of synergistic risks. Specifically, we developed two risk messages concerning the synergistic risk of developing esophageal cancer for individuals who consume both alcohol and tobacco. One message featured content concerning the biological mechanism by which alcohol and tobacco interact, and the other message featured probabilistic information concerning the likelihood of developing esophageal cancer for individuals who use alcohol and tobacco. Our principle aim was to identify which message content best helped individuals to understand that the combination presented a synergistic risk. More generally, our motivation for undertaking this study was to contribute to identifying specific communication approaches that may best help individuals improve their understanding of synergistic risks, so that they can make informed decisions and adopt appropriate behavioral responses.

\subsection{The Communication of Synergistic Risks}

The term 'synergistic risk' refers to a cause-and-effect concept. That is, the interaction of certain hazards initiates a mechanism (i.e., a cause), which results in a risk magnitude (i.e., an effect) that is greater than the sum of the risk magnitude attributable to each hazard when operating separately. Despite featuring these two components, empirical studies concerning the communication of synergistic risks have primarily focused on assessing messages that convey only the 'effect' component. Specifically, the messages have depicted probabilistic 
risk information for both the hazard combination and each of the constituent hazards. The intention of these messages has been to encourage recipients to identify that the risk presented by the combination is greater than the sum of the risk presented by the constituents. For example, Hampson et al. employed this approach when using pie-charts and explanatory text to communicate the lung cancer risk for individuals who are exposed to tobacco smoke, radon, or both. ${ }^{(13)}$ Although slices of the pie-charts provided a proportional representation of the risk magnitude attributable to each constituent hazard and the combination, the findings indicated this approach failed to help individuals understand that the radon-tobacco combination presents a synergistic risk (see reference ${ }^{16}$ ). Similarly, French et al. used bargraphs and explanatory text to convey probabilistic information concerning the additive health risk attributable to one hazard combination and the synergistic health risk attributable to a different combination. ${ }^{(9)}$ The results suggested that the bar-graphs failed to help participants differentiate between the risks attributable to each combination. By contrast, Bonnin-Scaon et al. showed that a series of messages featuring probabilistic information can enable recipients to make relatively accurate judgments of synergistic risks. ${ }^{(14)}$ Bonnin-Scaon et al. used one-to-one training sessions to teach individuals the likelihood of developing esophageal cancer for various levels of alcohol and tobacco consumption. In the sessions, participants received iterative feedback concerning the accuracy of their initial and revised risk estimates. Bonnin-Scaon et al. posited that it was primarily this provision of feedback that increased the participants' risk estimate calibration. Despite the reported success of this approach, it should be noted that each participant's training lasted one hour or more, indicating that this approach lacks efficiency.

The efficacy of helping individuals to understand synergistic risks using messages that describe the underlying mechanism, remains, to the best of our knowledge, empirically untested. However, there are reasons to believe that such messages could prove effective. For 
instance, research concerning individual health hazards has indicated that antecedent-related information can help individuals formulate a mental model of what may cause one to develop a particular health problem and, consequently, to personalize the threat. ${ }^{(17,18)}$ In addition, research has shown that many individuals view probabilistic risk information as insufficient, preferring to focus on understanding the potential causes of risks and, consequently, the relevant preventative actions. ${ }^{(19-21)}$ Hence, in relation to synergistic risks, risk communications that impart knowledge of the causal mechanism could increase the plausibility and vividness of the process by which particular hazards interact. This could heighten the personal relevance of the risk, whilst also implicitly conveying how the avoidance/removal of one factor can prohibit the mechanism. Moreover, a study by Dawson, Johnson and Luke found that individuals who demonstrated an awareness of synergistic risks often expressed a belief that the increased risk was attributable to an underlying mechanism

(e.g., chemical reaction between two drugs). ${ }^{(22)}$ Hence, risk messages detailing the causal mechanisms may provide a promising approach for communicating synergistic risks.

To the best of our knowledge, the efficacy of communicating both the mechanistic and probabilistic components of a synergistic risk remains untested. Such communications would explicate the cause-effect process and, therefore, could help individuals appreciate how a particular synergistic risk arises and to understand the magnitude of the threat. This more 'complete' understanding of synergistic risk may help improve decisions regarding precautionary behaviors, as there would be less scope for individuals to misunderstand what initiates the mechanism and how likely it is that the adverse outcome will occur.

\subsection{Assessing the Effectiveness of Communications Concerning Synergistic Risk}

In light of the limitations in the extant literature, we conducted a study with the principle aim of assessing the relative effectiveness of communicating a synergistic health 
risk using a message containing either mechanistic- or probabilistic-related information, or both. We assessed each message relative to a control group and measured the extent to which each of these messages improved participants' risk model (i.e., sub-additive, additive, or synergistic risk) judgments and the accuracy of their risk magnitude estimates for the hazard combination. In addition, we made relative assessments of each message's affective impact and capacity to influence professed behavioral intentions, and we assessed the extent to which each message was understood and trusted.

\subsubsection{Alcohol, Tobacco and Esophageal Cancer}

In our study, all participants were presented with information regarding the synergistic risk of developing esophageal cancer for individuals who use both alcohol and tobacco. We selected the alcohol-tobacco combination for several reasons. First, this synergistic risk is well-evidenced in several epidemiological studies. ${ }^{(1,23,24)}$ Second, several authors have expressed support for a hypothesized biological mechanism underling the synergy, ${ }^{(25-27)}$ and this hypothesis has been upheld by evidence from several bio-physics studies. ${ }^{(28-30)}$ Specifically, consumed alcohol acts as a solvent that lines the mucosa of the aero-digestive organs, and this increases the extent to which carcinogenic toxins in tobacco stick to and penetrate the tissue of these organs. Hence, the mechanism for this hazard combination is reasonably well understood and could be explained to lay individuals. Third, smoking and alcohol consumption are prevalent activities in many countries and research suggests that many individuals engage in both behaviors. ${ }^{(31-33)}$ Finally, smoking and alcohol consumption are two of the leading risk factors for esophageal cancer and less than one in ten people survive for ten years following diagnosis. ${ }^{(34)}$ Thus, the risk of esophageal cancer for individuals who both smoke and drink remains a critical public health issue. Consequently, this is an important synergistic health risk and there is information (regarding both the causal 
mechanism and risk magnitude) available for dissemination which could potentially influence decisions and behaviors.

\subsubsection{Communication Format and Contents}

The probabilistic data we presented to participants was conveyed via three icon arrays' (a.k.a., population figures or pictograms). Each array comprised a 10 x 10 grid of square-shaped icons with a white/black square representing an individual unaffected/affected by esophageal cancer. We employed icon arrays as this form of graphical analogy has been shown to be effective in (a) illustrating the proportional relationship between the numerator (i.e., those affected by the disease) and the denominator (i.e., the population), (b) increasing the accuracy of risk magnitude judgments, (c) facilitating efficient processing of probabilistic data, and (d) communicating risk magnitudes to individuals with varying numerical proficiency. ${ }^{(35-37)}$ We used separate icon arrays to convey the frequency with which the following develop esophageal cancer: (i) smokers (ii) drinkers, and (iii) those who are both smokers and drinkers. All three arrays were presented to participants simultaneously, with the intention of enabling them to identify that the risk presented by the alcohol-tobacco combination is greater than the sum of the risk presented by either substance alone. Each array was supported by explanatory text.

The information presented to participants concerning the biological mechanism underlying the alcohol-tobacco synergy was based on the results of the studies discussed above. Details of the mechanism were conveyed using three simplified pictorial diagrams, accompanied by explanatory text, depicting the esophagus during exposure to alcohol and tobacco (see section 2.2. for details). This format provided some parity with the format employed in the 'probabilistic condition' (i.e., three graphical images with supporting text), and research has shown that pictures accompanied by explanatory text are effective in 
helping individuals to develop mental models and a deep comprehension of concrete processes. ${ }^{(38,39)}$ Furthermore, these studies also show that simplified diagrams can help individuals to generate inferences that facilitate comprehension of the concepts depicted in the diagrams. Hence, it appears plausible that our diagrams depicting the biological mechanism could aid the generation of an inference that the interaction would dramatically increase the risk of esophageal cancer. More specifically, it is possible that the diagrams alone could enable individuals to infer that the risk is synergistic.

\subsubsection{Risk Comprehension and Numeracy}

The ability to understand and process probabilistic and mathematical concepts (a.k.a., numeracy) has been shown to vary significantly between individuals, ${ }^{(40,41)}$ and inadequate numerical skills can inhibit the comprehension of numeric risk information. ${ }^{(41-43)}$ Consequently, we also assessed whether participants' numerical skills affected their ability to identify, from the messages presented, the risk model and risk magnitude attributable to the alcohol-tobacco combination. If numeracy skills were found to moderate comprehension of this synergistic risk, it could be indicative of a weakness in our messages' capacity to facilitate comprehension amongst individuals of differing levels of numeracy.

\section{METHOD}

\subsection{Participants}

One-hundred-and-twenty-seven postgraduate management students $(57$ men, 70 women) aged 20 to $36(M=24.63, S D=3.11)$ participated in this study during a lecture at a UK university in 2011. We considered the use of a student sample appropriate because extant studies indicate that student samples tend to provide risk judgments for combined hazards that are consistent with the judgments provided by non-student samples such as alcoholics, 
homeowners, hospital patients, and community volunteers (for a review see reference ${ }^{9}$ ). Specifically, both groups tend to judge synergistic risks as either sub-additive or additive risks. Furthermore, extant research also indicates that an individual's educational background does not moderate the individual's understanding of an intervention concerning synergistic risks. ${ }^{(44)}$ Hence, we considered there to be sound reasons for concluding that the use of a student sample in our study would not affect the generalizability of our results.

\subsection{Materials}

Participants reviewed one of four A4-sized booklets, each of which contained the following opening statement: "Research evidence shows that regularly drinking alcohol can increase the chance of developing cancer of the esophagus (the food-pipe that links the mouth to the stomach). Research evidence also shows that regularly smoking tobacco can increase the chance of developing cancer of the esophagus." All booklets contained a message stating that within the booklet the terms 'heavy smoker' and 'heavy drinker' referred, respectively, to a person who (a) smokes more than 25 cigarettes per day, and (b) consumes the equivalent of two standard size bottles of wine per day.

One version of the booklet (control condition) contained no further information than that described above. Hence, participants in this condition were only made aware that using alcohol or tobacco increases the risk of esophageal cancer. They did not receive any information concerning the increased (i.e., synergistic) risk from using both substances.

The second version of the booklet (mechanism condition) provided participants with additional information, in the form of text and pictorial diagrams, describing the hypothesized mechanism that underlies the increased risk of developing esophageal cancer for people who both smoke and drink. The text read: "Research evidence shows that when a person drinks alcohol and smokes tobacco, the alcohol acts like a glue that causes the toxins in the smoke 
to stick to the moist tissue lining of the esophagus. The toxins are then more able to penetrate the tissue in the esophagus. This increases the chance of developing cancer in this area of the body. The three diagrams on the following page illustrate the process described above". Participants then reviewed three diagrams illustrating the mechanism (see Figure 1). Each diagram was $82 \mathrm{~cm} \times 53 \mathrm{~cm}$, printed in grey-scale, and accompanied by one sentence describing the process depicted in the diagram. Hence, participants in this condition were informed that the risk of developing esophageal cancer increases for individuals who both smoke and drink, but were not informed of the magnitude of the increase.

[Insert Figure 1 about here]

The third booklet (probabilistic condition) provided participants with information in the form of text and pictorial diagrams. In this version, the text read: "On the following page you will see three diagrams. Each of the three diagrams represents a population of 100 people. Each square represents one person. A black square represents a person who will develop esophageal cancer during their lifetime. The data in these diagrams is based on the results of scientific research." Participants then reviewed three icon arrays which each contained 100 squares (see Figure 2). In the first two icon arrays, two and six squares, respectively, were shown in black (to the left of the bottom row) and the arrays were accompanied by the sentences: "A population of 100 heavy smokers" and "a population of 100 heavy drinkers", respectively. The third array showed twenty-nine squares in black across the bottom three rows, with all other squares in white. Beneath this array was the sentence: "A population of 100 people, each one of whom is both a heavy smoker and a heavy drinker." Each array was $47 \mathrm{~m} \times 47 \mathrm{~cm}$, and printed in black-and-white. The three arrays were accompanied by a 'key' indicating that a white/black square represented one person who would not/would develop esophageal cancer. Hence, participants in this condition were only provided with probabilistic information (in pictorial form) from which they could infer that 
the alcohol-tobacco combination presented a greater risk of developing esophageal cancer than the sum of the risk for either substance alone.

\section{[Insert Figure 2 about here]}

The additional information provided in the fourth booklet included identical versions of that featured in both the mechanism condition and in the probabilistic condition. The mechanism-related information/diagrams were presented first, followed by the sentence "The extent to which the harmful interaction between alcohol and tobacco increases the chance of developing esophagus cancer is represented in the three diagrams below". The probabilityrelated information/diagrams were then presented. Thus, participants in this condition, consistent with the chronology of the cause-effect process, first reviewed information that explained the mechanism and then the probabilistic/synergistic risk.

The participants in all four conditions read the assigned information booklet and, at their own pace, then immediately completed the same questionnaire. They were instructed not to look in the informational booklet whilst completing the questionnaire (compliance with this instruction was monitored by the experimenters and no breaches were observed). The questionnaire commenced with the following paragraph: "Research evidence shows that a person who is a heavy smoker has a 2 in 100 chance of developing esophageal cancer during their lifetime. Research evidence also shows that a person who is a heavy drinker has a 6 in 100 chance of developing esophageal cancer during their lifetime." Hence, all participants were made aware of the likelihood of developing esophageal cancer for individuals who are either heavy smokers or heavy drinkers only. Following this paragraph, participants were asked to consider the likelihood of an individual developing esophageal cancer if that individual was both a heavy smoker and a heavy drinker. They were asked to state, using a multiple-choice response format, whether they judged this likelihood to be "less than", "equal to", or "more than" the likelihood for an individual who is a heavy smoker only, 
"added to" the likelihood for an individual who is a heavy drinker only. The layout, style, and content of this judgment task were developed in accordance with guidelines outlined by Osterlind for the construction of multiple-choice response items. ${ }^{(45)}$ Participants were then presented with a second judgment task which asked: "Out of a population of 100 people, each of whom is a heavy smoker and a heavy drinker, how many of them do you estimate will develop esophageal cancer during their lifetime?"

In the subsequent part of the questionnaire, participants used Visual Analogue Scales (VAS), numbered 1 to 7 , to state how worried ('not at all worried' = 1, 'extremely worried' = 7) and frightened ('not at all frightened' $=1$, 'extremely frightened' $=7$ ) he/she would be about developing esophageal cancer during their lifetime if he/she were both a heavy smoker and a heavy drinker. Participants also used seven-point VASs to state how likely ('not at all likely' $=1$, 'extremely likely' $=7$ ) it is that, based on the information he/she read in the booklet, he/she would (a) quit smoking, (b) quit drinking, (c) reduce smoking, and (d) reduce drinking. Participants were asked to make all responses to these questions 'as if he/she was' a heavy smoker and drinker, because it was anticipated that a majority of participants would probably not be both heavy smokers and drinkers (consistent with this prediction, $94 \%$ of our participants reported either smoking only [at least once-a-day], drinking only [at least once-aweek], or doing neither).

Finally, participants used seven-point VASs to describe the extent to which they understood ('not at all easy to understand' $=1$, 'extremely easy to understand' $=7$ ) and trusted ('not at all trustworthy' $=1$, 'extremely trustworthy' $=7$ ) the information in the booklet.

\subsection{Measurement of Numeracy}


Participants completed eight numerical questions established by Lipkus, Samsa and Rimer. ${ }^{(40)}$ For familiarization, participants initially read an example question (with the correct answer provided) prior to completing the questions. An example of one of the eight questions reads "Which of the following numbers represents the biggest chance of getting a disease? 1 in 100, 1 in 1000, 1 in 10?" The fifth question comprised two sub-questions ("If the chance of getting a disease is 10\%, how many people would be expected to get the disease: (a) out of 100?, and (b) out of 1000?), which we treated as two separate items. Hence, participants could achieve a maximum score of nine.

\subsection{Design}

The study followed a 4 (risk communication: control $[n=30]$, mechanism $[n=32]$, probabilistic $[n=28]$, mechanism+probabilistic $[n=37])$ x 2 (numeracy: more, less) between-subjects design. The two principal dependent variables were (a) risk model judgments (sub-additive, additive, or synergistic) and (b) frequency estimates (e.g., 29 out of 100).

\subsection{Procedure}

Approximately 40 copies of each of the four booklets were manually shuffled into one pile and then distributed amongst the participants. Participants were informed that they had unlimited time to review the booklet and to complete the questionnaire. Participants were advised not to consult any other resources or persons during the experiment. The procedure was supervised by three experimenters. It was observed that participants took between nine and 20 minutes to review the booklet and complete the questionnaire (the questionnaire contained the judgment tasks, VAS questions, numeracy scale and requests for sociodemographic data). 


\subsection{Analysis}

Hierarchical loglinear analysis was employed to test for main effects and interactions between categorical variables, and significant results were further analyzed using chi-square $\left(\chi^{2}\right)$ tests. Analyses of variance (ANOVAs) were employed to assess the influence of risk messages on continuous dependent variables (e.g., VAS responses), and significant differences were further analyzed using Bonferroni post hoc tests.

\section{RESULTS}

\subsection{Numeracy of Participants}

The mean numeracy score was $7.15(S D=1.73 ; M d=7)$, and scores ranged from 1 to 9. Participants were split into two groups (more or less numerate) according to the group median score. Hence, $48.8 \%(n=62)$ of participants with 8 or 9 items correct were classified as more numerate, and $51.2 \%(n=65)$ of participants with 7 or fewer correct items were classified as less numerate (for similar procedures see references ${ }^{35,42,46}$ ). As observed here, on extant numeracy scales it is commonplace to obtain a skewed distribution of scores where the observed median score is higher than the mid-score on the scale. ${ }^{(47)}$ There was no significant difference in the proportion of more and less numerate participants in each of the four risk communication conditions, $\chi^{2}(3)=3.04, p>0.39$.

\subsection{Data Preparation}

Categorical risk model judgments for each of the four risk communication groupings are displayed in Table I. To determine whether three-way loglinear analyses (e.g., risk model $\mathrm{x}$ risk communication $\mathrm{x}$ numeracy) could be performed, we assessed whether the data met the test assumption that no less than $20 \%$ of the cells should have expected frequencies less than 
5. ${ }^{(48)}$ As $67 \%$ of cells featured expected frequencies less than 5 , the data did not meet this assumption. To address this issue, the 'sub-additive' and 'additive' categories were collapsed into one category, resulting in two categories for the risk model variable i.e., 'synergistic' and 'non-synergistic' (for clarity, Figure 3 shows the risk model judgments in these collapsed categories). The data then met the test assumptions, and the three-way analysis was viable. Collapsing categories into dichotomous groups is appropriate for a hierarchical loglinear analysis where the research aims are not affected. ${ }^{(48)}$

[Insert Table I about here]

[Insert Figure 3 about here]

\subsection{Risk Model Judgments, Risk Communication, and Numeracy}

To assess whether participants' risk model judgments varied according to risk communication and/or numeracy, we performed a three-way loglinear analysis: risk model $\mathrm{x}$ risk communication $\mathrm{x}$ numeracy. This identified a significant main effect for risk model, $\chi^{2}$ $(1)=6.68, p=0.01$. The main effect for risk model was attributable to the greater proportion of participants, across the whole sample, who judged that the combination would present a synergistic risk $(61 \%)$ rather than a non-synergistic risk $(39 \%), \chi^{2}(1)=6.62, p<0.01$. Furthermore, the main effect for risk model was qualified by a two-way interaction between risk model and risk communication, $\chi^{2}(3)=9.90, p<0.05$. No other significant interactions or main effects were found, $\chi^{2} \mathrm{~s}(3)<2.68, p \mathrm{~s}>0.05$. Hence, participants' risk judgments did not differ significantly according to numerical ability, and this was the case irrespective of the type of risk communication that participants reviewed.

To investigate the identified two-way interaction between risk model and risk communication, chi-square analyses of participants' risk model judgments were performed for each risk communication grouping. To address the increased risk of a Type I error when 
performing these multiple comparisons, a Bonferroni correction $(p=0.05 / 4=0.0125)$ was employed. In the control condition, there was no significant difference, $\chi^{2}(1)=0.53, p>$ 0.05 , between the proportion of participants who judged that the hazard combination would present a synergistic risk (43\%) and a non-synergistic risk (57\%). Similarly, there was no significant difference between these two proportions in (a) the mechanism condition (53\% synergistic risk, $47 \%$ non-synergistic risk), $\chi^{2}(1)=0.13, p>0.05$, or (b) the probabilistic condition (68\% synergistic risk, $32 \%$ non-synergistic risk), $\chi^{2}(1)=3.57, p>0.05$. However, in the mechanism+probabilistic condition, a significantly greater proportion of participants (78\%) judged that the combination would present a synergistic risk (cf. non-synergistic risk), $\chi^{2}(1)=11.91, p<0.001$

Chi-square tests were employed to assess whether the risk model judgments made by participants in the (i) mechanism (ii) probabilistic or (iii) mechanism+probabilistic conditions differed significantly from those in the control condition. A Bonferroni correction was employed $(p=0.05 / 3=0.017)$. The results revealed that the participants' risk model judgments in the mechanism condition and the probabilistic condition did not differ significantly from participants' judgments in the control condition, $\chi^{2} \mathrm{~s}(1)<3.53, p \mathrm{~s}>0.05$. By contrast, a significantly greater proportion of participants in the mechanism+probabilistic (cf. control) condition judged that the hazard combination would present a synergistic risk, $\chi^{2}$ $(1)=8.70, p<0.01$.

\subsection{Accuracy of Frequency Estimates, Risk Communication, and Numeracy}

As described above, participants were asked to estimate how many individuals, each of whom is a heavy smoker and a heavy drinker, would develop esophageal cancer during their lifetime. Calculations based on data reported by Cancer Research UK and Zambon et al. indicate that 29 of 100 such individuals will develop esophageal cancer in their lifetime. ${ }^{(24,49)}$ 
The accuracy of participants' frequency estimates was assessed in relation to this statistic. The distribution of participants' estimates, which ranged from 1 to 90 , demonstrated a peak from 27 to 31, followed by a drop in responses beyond these limits. This indicated participants either did not know the correct frequency, or knew the correct frequency within $\pm 2 \%$. Thus, the frequency estimate variable was transformed into a dichotomous categorical variable (accuracy: accurate, inaccurate), with estimates from 27 to 31 deemed to be accurate, and all other estimates deemed inaccurate. For a similar approach to assessing the accuracy of risk/frequency judgments see Galesic et al. ${ }^{(35)}$

Categorical frequency estimates for each of the four risk communication groupings are displayed in Figure 4. A three-way loglinear analysis (accuracy $\mathrm{x}$ risk communication $\mathrm{x}$ numeracy) was performed to assess whether participants' estimates varied according to risk communication and/or numeracy. ${ }^{1}$ This identified a significant main effect for accuracy, $\chi^{2}$ $(1)=20.75, p<0.001$. The main effect for accuracy was attributable to the greater proportion of participants, across the whole sample, who provided inaccurate $(70 \%)$ rather than accurate $(30 \%)$ frequency estimates, $\chi^{2}(1)=20.16, p<0.001$. Furthermore, the main effect for accuracy was qualified by a two-way interaction between accuracy and risk communication, $\chi^{2}(3)=49.42, p<0.001$. However, no other significant interactions or main effects were found, $\chi^{2} \mathrm{~s}(3)<4.00, p \mathrm{~s}>0.05$. Hence, the accuracy of participants' frequency estimates did not differ significantly according to numerical ability and this was the case irrespective of the type of risk message reviewed. ${ }^{2}$

[Insert Figure 4 about here]

To investigate the identified two-way interaction between accuracy and risk communication, chi-square analyses of participants' frequency estimates were performed for each risk communication grouping. A Bonferroni correction ( $p=0.05 / 4=0.0125)$ was employed. For the control condition, the test could not be computed as $100 \%$ of participants 
provided inaccurate frequency estimates. Similarly, 93\% of participants in the mechanism condition provided inaccurate estimates and, consequently a significant difference, $\chi^{2}(1)=$ 22.53, $p<0.001$, was observed between the proportion of accurate and inaccurate estimates. Separate tests for the probabilistic and mechanism+probabilistic conditions both revealed no significant difference, $\chi^{2} \mathrm{~s}(1)<0.94, p s>0.05$, between the proportion of accurate and inaccurate estimates in each condition (probabilistic/mechanism+probabilistic condition: $59 / 51 \%$ accurate, $41 / 49 \%$ inaccurate).

Tests were performed to examine whether the accuracy of participants' frequency estimates in the mechanism, probabilistic, and mechanism+probabilistic conditions differed from participants' estimates in the control condition. A Bonferroni correction was employed $(p=0.05 / 3=0.017)$. The results revealed that the accuracy of participants in the control and mechanism conditions did not differ significantly, $\chi^{2}(1)=2.07, p>0.05$. By contrast, a significant difference was identified between the accuracy of participants' estimates in the control and (i) the probabilistic condition, $\chi^{2}(1)=24.72, p<0.001$, and (ii) the mechanism+probabilistic condition, $\chi^{2}(1)=21.50, p<0.001$. That is, participants in the probabilistic and the mechanism+probabilistic conditions provided a significantly greater proportion of accurate risk estimates than participants in the control condition.

\subsection{Affective Responses, Behavioral Intentions, Comprehension, and Trust}

For each of the four risk communication conditions, participants' mean responses on the seven-point VASs are displayed in Table II. Independent measures ANOVAs were performed to assess whether participants' responses varied according to the conditions. This identified that there was no significant difference between the four conditions in terms of (i) the extent to which the information in each booklet was trusted $(M=4.67, S D=1.50)$ and understood $(M=5.76, S D=1.26), F \mathrm{~s}(3,123)<1.38, p \mathrm{~s}>0.05$, (ii) affective evaluations, 
such as worry $(M=5.15, S D=1.54)$ and fright $(M=4.74, S D=1.68), F \mathrm{~s}(3,123)<2.02, p \mathrm{~s}>$ 0.05 , and (iii) the likelihood of quitting drinking $(M=3.89, S D=1.79)$, reducing drinking $(M$ $=4.85, S D=1.82)$, or reducing smoking $(M=5.07, S D=1.77), F \mathrm{~s}(3,123)<1.59 p \mathrm{~s}>0.05$. However, the likelihood of quitting smoking did differ significantly between the risk communication conditions, $F(3,123)=3.27, p<0.05$. A Bonferroni post hoc test $(p=0.05 / 4$ $=0.0125)$ revealed that participants in the mechanism condition $(M=4.53, S D=1.87)$ were significantly ( $p=0.04$ ) more likely to report they would quit smoking (cf. participants in the control condition) if they were both a heavy smoker and a heavy drinker $(M=3.30, S D=$ 1.56); no other significant ( $p \mathrm{~s}>0.05$ ) pairwise comparisons were identified.

[Insert Table II about here]

\section{DISCUSSION}

Our results show that, in relation to the combined use of alcohol and tobacco, messages which convey evidence-based details of the underlying causal mechanism, the resultant probabilistic risk, or both of these, each has its own strengths in helping individuals to understand that the combination presents a synergistic risk. This suggests that each of these message contents should be selected according to the communicator's objectives. Specifically, we found that the message concerning both the causal mechanism and the resultant risk magnitude was the only approach that led to a significant improvement in the veridicality (i.e., consistent with empirical data) of individuals' risk model judgments. This suggests that, where the principle aim is to help individuals understand that the hazard combination presents a synergistic risk, the communicator should consider conveying details of both components. We also found that the provision of probabilistic information, either alone or in conjunction with mechanistic information, can lead to significant improvements in the extent to which individuals accurately understand the risk magnitude attributable to a 
particular hazard combination. This indicates that messages detailing the probabilistic risk are most likely to be appropriate when the communicator's sole objective is to help impart knowledge of the synergistic risk magnitude. Furthermore, our study suggests that a message detailing only the causal mechanism of the synergistic risk may be most effective in influencing protective behavioral intentions. That is, a message which focuses the recipient's attention on the actual cause of the synergistic risk may help that person recognize that the threat posed by the hazard combination can be reduced/eliminated by the removal of one of the hazards. Furthermore, the absence of variation in affective reactions (i.e., worry and fright) between the four conditions indicates that evidence-based messages concerning the synergistic risk may be particularly appropriate when the communicator's motive is to facilitate informed decision-making rather than to arouse fear or provoke disproportionate precautionary behaviors. However, it should be noted that research evidence also suggests that the adoption of health related behaviors often follows an inversed U-shaped pattern as worry/perceived risk increases. ${ }^{(53,54)}$ Therefore, the absence of affective reactions in our study could suggest that our intervention may not provoke a magnitude of worry/fear that is sufficient to motivate behavioral changes. Nonetheless, a balance is clearly required in the provocation of affective reactions because additional studies indicate, consistent with the inverse U-shaped pattern, that heightened fear can lead to a sense of helplessness and/or reluctance to adopt self-protective behaviors. ${ }^{(55,56)}$

Beyond the key findings mentioned above, there are additional aspects of our results that require further consideration and which provide useful insights into the communication of synergistic risks. First, the frequency estimates of participants in the two probabilistic conditions (cf. control condition) show that the icon arrays helped just over half of them to acquire precise knowledge of the risk magnitude. On one hand, this finding shows that, in contrast to the results of previous studies that have not employed icon arrays, ${ }^{(9,13)}$ 
probabilistic information about synergistic risks can be effectively and efficiently conveyed using visual aids. This relative success is consistent with other studies that have demonstrated the capacity of icon arrays to substantially improve the accuracy of risk estimates. ${ }^{(35,36)}$ On the other hand, just less than half of the participants in our two probabilistic conditions did not make accurate risk estimates for the alcohol-tobacco combination. This highlights a potential limitation of the icon array approach which, according to our findings, cannot be attributed to differences in numerical ability. Hence, there is further scope for identifying (a) how probabilistic information concerning synergistic risk magnitudes may be conveyed with greater effectiveness and (b) what factors may moderate the comprehension of probabilistic information concerning synergistic risks.

Second, previous studies indicate that individuals are typically (a) interested to know what causes particular [health] problems because this can help them to identify what preventative action they could take, and (b) more likely to make behavioral decisions based on case-based, concrete information as opposed to statistical, abstract information (see reference ${ }^{17}$ ). This could explain why participants in our mechanism condition (cf. control condition) reported being significantly more likely to quit smoking (i.e., the participants identified that removing tobacco inhibits the harmful synergy). However, this interpretation then presents two important questions. The first question is why did the mechanism message influence participants to report a greater likelihood of quitting smoking but not quitting drinking? We suggest that, whilst participants could identify that the removal of either substance would prohibit the harmful synergy, the preference for quitting smoking may be largely motivated by the common perception tobacco smoking is wholly deleterious to health, often to the extent that individuals over-estimate the harmful effects. ${ }^{(57,58)}$ By contrast, research suggests that alcohol consumption is perceived as less risky than smoking and has some health benefits. ${ }^{(59,60)}$ The second question raised is why the participants in the 
mechanism+probabilistic condition (cf. control condition) did not also report being more likely to quit drinking? We speculate that this may have been because the probabilistic information attenuated the impact of the mechanism information. In particular, a 'recencyeffect' may have been elicited in which the latter part of the message became dominantly salient in the minds of the participants, reducing the impact of the mechanism information. ${ }^{(61)}$ Equally, the probabilistic information may have served to decrease the perceived threat posed by the alcohol-tobacco interaction, because the icon array may have been effective at highlighting that the proportion of heavy smokers and drinkers unaffected by esophageal cancer was larger than the affected proportion; similarly, other scholars have noted that communication formats which effectively highlight both the affected and unaffected populations in this manner may lead to a decrease in perceived risk. ${ }^{(62,63)}$ Whatever the cause of this finding, our results suggest that those wishing to communicate information about synergistic risks using mechanistic+probabilistic message contents should be mindful of how latter aspects of the message may impact upon the effects of previous aspects.

Third, the concept of 'synergistic risk' is quantitatively defined (i.e., the risk attributable to the hazard combinations exceeds the sum of the risk attributable to each constituent hazard). Hence, it would seem reasonable to suggest that individuals with lower numerical skills may be less adept at understanding this particular risk concept. However, in contrast to this proposition, our results indicate that an individual's numerical ability does not necessarily moderate whether he/she understands, either pre or post intervention, that a hazard combination presents a synergistic risk. Consistent with the findings of Bonnin-Scaon et al. and Hampson, Andrews, Barckley, Lee and Lichtenstein, our study suggests that such an understanding is moderated by access to opportunities to effectively learn about the interaction of the hazards and the resultant synergistic effects, rather than to the unique characteristics of the individual. ${ }^{(14,64)}$ Importantly, this highlights that the 
mechanism+probabilistic message employed in our study may represent part of a valuable model of how many individuals could be helped to make better informed decisions about the risk attributable to combined hazards.

Fourth, we suggested above (see Section 1.2.2.) that it was possible that the diagrams of the biological mechanism alone may enable individuals to infer that a synergistic risk model is attributable to the alcohol-tobacco combination. However, our results do not support this proposition. This suggests that, whilst previous studies show that simplified diagrams alone can aid the generation of inferences, the participants in the mechanism condition may have lacked the necessary knowledge to infer that the mechanism increases the risk synergistically. More specifically, the diagrams alone may have helped the participants to understand that the mechanism increases the risk of esophageal cancer, but did not provide sufficient information to help our participants to understand that the increase in risk is greater than the sum of the risk attributable to the two substances operating alone. This interpretation of our findings is supported by evidence from Moravcsik and Kintsch who found that the accuracy of the inferences that individuals can draw from educational materials is often influenced by additional knowledge possessed by the learner. ${ }^{(65)}$

Finally, our messages only conveyed details of the synergistic risk of developing esophageal cancer for smokers/drinkers. Consequently, it could be argued that the relative effectiveness of our mechanism+probabilistic message may not be replicable for messages concerning other hazard combinations that also present synergistic risks. However, our results suggest that the effectiveness of combining the mechanistic and probabilistic messages, compared to using either of these messages alone, is attributable to its capacity to enable individuals to specifically identify that there is a relationship between the 'cause' and 'effect' components respectively depicted in each message. As there appears to be little reason to conclude that the ability to identify such a relationship is specific to the likelihood 
of developing esophageal cancer for people who use alcohol and tobacco, it seems reasonable to assert that the relative effectiveness of our mechanism+probabilistic message should be replicable for other hazard combinations. In support of this argument, research evidence from Butcher and Kintsch indicates that educational materials, which use diagrams and supporting text to communicate technical concepts, are particularly effective at facilitating holistic comprehension via the integration of separate inferences drawn from multiple information sources. ${ }^{(38,66)}$ Furthermore, research also shows that the ability to achieve holistic comprehension from multiple messages featuring diagrammatic/pictorial representations can occur across a range of disciplines, such as engineering and human anatomy, and, therefore, this ability does not appear to be domain- and/or content-specific. ${ }^{(38,67)}$

\subsection{Limitations and Future Directions}

Our study has some limitations that could be addressed in future research. First, whilst there are legitimate reasons to believe that our use of a well-educated sample does not limit the generalizability of our findings to other populations (see section 2.1) we would still encourage researchers to conduct future studies that assess the effectiveness of our messages using a variety of populations. This may help to identify whether there are any individual or socio-demographic characteristics (which currently remain undetected by extant research) that may moderate the comprehension of synergistic risks. Similarly, because our sample consisted mainly of individuals who were not smokers and drinkers we acknowledge that (a) we cannot determine whether our messages would influence the behavioral intentions of individuals who do smoke and drink, and (b) the sample (cf. smokers and drinkers) may have been less likely to demonstrate a strong affective reaction to our messages. Second, we presented our messages using specific visual formats because extant studies have highlighted the efficacy of these particular approaches. However, we acknowledge that other formats 
could prove more effective and/or efficient and that this is something future studies could assess. For example, researchers might explore whether the efficiency of Bonnin-Scaon et al.'s intervention could be improved by using a single interactive computer-based chart to communicate risk, rather than manually presenting numerous cue-configuration cards to individuals across several training sessions. Third, we suggested above that a 'recency effect' may explain why there was no effect of the mechanism information on likelihood of quitting when followed by probabilistic information. A future study could reverse the order of the presentation of probabilistic and antecedent information to test this proposition. Finally, the risk messages we employed were designed to empirically assess the extent to which each specific message would enable the recipient to infer that the combination presents a synergistic risk. Therefore, it is promising to recognize that the efficacy of these messages may be further enhanced by additional information, which provides more explicit information concerning the fundamental purpose of the communication.

\section{CONCLUSION}

Our study has addressed a need to identify message content that is both effective and efficient in helping individuals to understand a synergistic risk. Importantly, our results highlight the specific merits and potential weaknesses of using messages that convey information about the mechanistic and/or probabilistic components of this risk concept. Specifically, messages that explain both components may provide the most effective approach for enabling individuals to understand both the risk model and magnitude attributable to specific hazard combinations. Furthermore, in comparing our results with those obtained in previous studies, it appears that the particular visual format employed may also influence the effectiveness of messages that convey probabilistic information about synergistic risks. Our findings can be utilized in the development of a general model for 
communications concerning synergistic risks and, therefore, can contribute to improving the important risk-related decisions that individuals make concerning combined hazards. However, further research is still needed to explore alternative approaches that can help individuals to understand the increasing number of synergistic risks that are being identified by the scientific community. 


\section{REFERENCES}

1. Lee CH, Lee JM, Wu DC, Hsu HK, Kao EL, Huang HL, Wang TN, Huang MC, Wu MT. Independent and combined effects of alcohol intake, tobacco smoking and betel quid chewing on the risk of esophageal cancer in Taiwan. International Journal of Cancer, 2005; 113: 475-482.

2. Talamini R, Bosetti C, La Vecchia C, Dal Maso L, Leiv F, Bidoli E, Negri E, Pasche C, Vaccarella S, Barzan L, Franceschi S. Combined effect of alcohol and tobacco on laryngeal cancer risk: A case-control study. Cancer Causes and Control, 2002; 13: 957-964.

3. Delaney JA, Opatrny L, Brophy JM, Suissa S. Drug-drug interactions between antithrombotic medications and the risk of gastrointestinal bleeding. Canadian Medical Association Journal, 2007; 117: 347-351.

4. van Os J, Pedersen CB, Mortensen PB. Confirmation of synergy between urbanicity and familial liability in the causation of psychosis. American Journal of Psychiatry, 2004; 161: $2312-2314$.

5. Drake BG, Hughes L, Johnson EA, Seibel BA, Cochrane MA, Fabry VJ, Rasse D, Hannah L. Synergistic Effects. In: Lovejoy, T.E., \& Hannah, L.E., editors. Climate Change and Biodiversity. Michigan: Yale University Press; 2005. p. 296-316.

6. Ben Q, Cai Q, Li Z, Yuan Y, Ning X, Deng S, Wang K. The relationship between newonset diabetes mellitus and pancreatic cancer risk: A case-control study. European Journal of Cancer, 2001; 47: 248-254.

7. Takahashi M, Kimura A. HLA and CTLA4 polymorphisms may confer a synergistic risk in the susceptibility to Graves' disease. Journal of Human Genetics, 2010; 55: 323326. 
8. Dawson IGJ, Johnson JEV, Luke MA. Do people believe combined hazards can present synergistic risks? Risk Analysis, 2011b; doi: 10.1111/j.1539-6924.2011.01693.x

9. French DP, Sutton S, Kinmonth AL, Marteau TM. Assessing perceptions of risks due to multiple hazards. Journal of Risk Research, 2006; 9: 657-682.

10. Leiserowitz A. Climate change risk perception and policy preferences: The role of affect, imagery, and values. Climate Change, 2006; 77: 45-72.

11. Berenbaum MC. What is Synergy? Pharmacological Reviews, 1989; 41: 93-141.

12. Cogliano VJ. Plausible upper bounds: Are their sums plausible? Risk Analysis, 1997; 17: $77-84$.

13. Hampson SE, Andrews JA, Lee ME, Foster LS, Glasgow RE, Lichtenstein E. Lay understanding of synergistic risk: the case of radon and cigarette smoking. Risk Analysis, 1998; 18: 343-350.

14. Bonnin-Scaon S, Lafon P, Chasseigne G, Mullet E, Sorum PC. Learning the relationship between smoking, drinking alcohol and the risk of esophageal cancer. Health Education Research, 2002; 17: 415-424.

15. Lipkus IM. Numerical, verbal, and visual formats of conveying health risks: suggested best practices and future recommendations. Medical Decision Making, 2007; 27: 696713.

16. Lipkus IM, Hollands JG. The visual communication of risk. Journal of the National Cancer Institute: Monographs, 1999; 25: 149-163.

17. Rothman AJ, Kiviniemi MT. Treating people with information: An analysis and review of approaches to communicating health risk information. Journal of the National Cancer Institute: Monographs, 1999; 25: 44-51.

18. Sherman SJ, Cialdini RB, Schwartzman DF, Reynolds KD. Imagining can heighten or lower the perceived likelihood of contracting a disease: The mediating effect of ease 
of imagery. Personality and Social Psychology Bulletin, 1985; 11: 118-127.

19. Boeije HR, Janssens CJW. It might happen or it might not: How patients with multiple sclerosis explain their perceptions of prognostic risk. Social Science and Medicine, 2004; 59: 861-868.

20. French DP, Hevey D. What do people think about when answering questionnaires to assess unrealistic optimism about skin cancer? A think aloud study. Psychology, Health \& Medicine, 2008; 13: 63-74.

21. Golding D, Sheldon S, Plough A. Evaluating risk communication: Narrative vs. technical presentation of information about radon. Risk Analysis, 1992; 12: 27-35.

22. Dawson IGJ, Johnson JEV, Luke MA. Subjective judgments of synergistic risks: A cognitive reasoning perspective. British Journal of Psychology, 2012; 103: 203-223.

23. Castellsague X, Munoz N, De Stefani E, Victora CG, Castelletto R, Rolon PA, Quintana MJ. Independent and joint effects of tobacco smoking and alcohol drinking on the risk of esophageal cancer in men and women. International Journal of Cancer, 1999; 82: 657-664.

24. Zambon P, Talamini R, La Vecchia C, Dal Maso L, Negri E, Tognazzo S, Simonato L, Franceschi S. Smoking, type of alcoholic beverage and squamous-cell esophageal cancer in northern Italy. International Journal of Cancer, 2000; 86: 144-149.

25. Blot WJ. Alcohol and cancer. Cancer Research, 1992; 52: 2119-2123.

26. Boffetta P, Hashibe M. Alcohol and cancer. Lancet Oncology, 2006 ; 7: 149-156.

27. Flanders WD, Rothman KJ. Interaction of alcohol and tobacco in laryngeal cancer. American Journal of Epidemiology, 1982; 115: 371-379.

28. Du X, Squier CA, Kremer MJ, Wertz PW. Penetration of N-nitrosonornicotine (NNN) across oral mucosa in the presence of ethanol and nicotine. Journal of Oral Pathology and Medicine, 2000; 29: 80-85. 
29. Howie NM, Trigkas TK, Cruchley AT, Wertz PW, Squier CA, Williams DM. Short-term exposure to alcohol increases the permeability of human oral mucosa. Oral Disease, 2001; 7: 349-354.

30. Squier CA, Cox P, Hall BK. Enhanced penetration of nitrosonornicotine across oral mucosa in the presence of ethanol. Journal of Oral Pathology and Medicine, 1986; 15: $276-279$.

31. World Health Organization. The world health report. Reducing risks, promoting healthy life. Geneva: World Health Organization, 2002.

32. Bien TH, Burge R. Smoking and Drinking: A Review of the Literature. Substance Use \& Misuse, 1990; 25: 1429-1454.

33. Dierker L, Lloyd-Richardson E, Stolar M, Flay B, Tiffany S, Collins L, Bailey S, Nichter M, Nichter M, Clayton R. The proximal association between smoking and alcohol use among first year college students. Drug and Alcohol Dependence, 2006; 81: 1-9.

34. Cancer Research UK. Fact Sheet: Oesophageal cancer survival statistics. http://info.cancerresearchuk.org/cancerstats/types/oesophagus/survival/, Accessed on December 2, 2011.

35. Galesic M, Garcia-Retamero R, Gigerenzer G. Using icon arrays to communicate medical risks: Overcoming low numeracy. Health Psychology, 2009; 28: 210-216.

36. Garcia-Retamero R, Galesic M. Who profits from visual aids: Overcoming challenges in people's understanding of risks. Social Science and Medicine, 2010; 70: 1019-1025.

37. Timmermans DRM, Ockhuysen-Vermey CF, Henneman L. Presenting health risk information in different formats: The effect on participants' cognitive and emotional evaluations and decisions. Patient Education and Counseling, 2008; 73: 443-447.

38. Butcher KR. Learning from text with diagrams: Promoting mental model development and inference generation. Journal of Educational Psychology, 2006; 98: 182-197. 
39. Glenberg AM, Langston WE. Comprehension of illustrated text: Pictures help to build mental models. Journal of Memory and Language, 1992; 31: 129-151.

40. Lipkus IM, Samsa G, Rimer BK. General performance on a numeracy scale among highly educated samples. Medical Decision Making, 2001; 21: 37-44.

41. Peters E. Numeracy and the perception and communication of risk. Annals of the New York Academy of Sciences, 2008; 1128: 1-7.

42. Dieckmann NF, Slovic P, Peters EM. The use of narrative evidence and explicit likelihood by decision-makers varying in numeracy. Risk Analysis, 2009; 29: 14731488.

43. Keller C, Siegrist M, Visschers V. Effect of Risk Ladder Format on Risk Perception in High- and Low-Numerate Individuals. Risk Analysis, 2009; 29: 1255-1264.

44. Condit CM, Shen L. Public Understanding of Risks from Gene-Environment Interaction in Common Diseases: Implications for Public Communications. Public Health Genomics, 2011; 14: 115-124.

45. Osterlind SJ. Constructing Test Items: Multiple-Choice, Constructed-Response, Performance, and Other Formats (2nd ed.). Boston: Kluwer Academic, 1998.

46. Keller C, Siegrist M. Effect of risk communication format on risk perception depending on numeracy. Medical Decision Making, 2009; 29, 483-490.

47. Peters E. Numeracy and Risk. Plenary session presentation delivered at the $20^{\text {th }}$ Society for Risk Analysis (Europe) Meeting, Stuttgart, Germany; June 2011.

48. Field A. Discovering Statistics Using SPSS (3rd ed.). London: SAGE Publications, 2009.

49. Cancer Research UK. Fact Sheet: Oesophageal cancer - UK incidence statistics. http://info.cancerresearchuk.org/cancerstats/types/oesophagus/incidence/, Accessed on December 2, 2011.

50. Dickert S, Kleber J, Peters E, Slovic, P. Numeracy as a precursor to pro-social behaviour: 
The impact of numeracy and presentation format on the cognitive mechanisms underlying donation decisions. Judgment and Decision Making, 2011; 6: 638-650.

51. Peters E., Hart PS, Fraenkel L. Informing Patients: The Influence of Numeracy, Framing, and Format of Side Effect Information on Risk Perceptions. Medical Decision Making, 2010; 31: 432-436.

52. Hayes AF. PROCESS: A versatile computational tool for observed variable moderation, mediation, and conditional process modelling, 2012; Manuscript submitted for publication.

53. Andrade EB. Behavioral Consequences of Affect: Combining Evaluative and Regulatory Mechanisms. Journal of Consumer Research, 2005; 32: 355-362.

54. Bowen DJ, Alfano CM, McGregor BA, Andersen MR. The relationship between perceived risk, affect, and health behaviors. Cancer Detection and Prevention, 2004; 28(6), 409-417.

55. Fischhoff B, Gonzalez RM, Lerner JSSDA. Evolving judgments of terror risks: foresight, hindsight, and emotion. Journal of Experimental Psychology: Applied, 2005; 11: 124139.

56. Weinstein ND, Lyon JE, Rothman AJ, Cuite CL. Preoccupation and affect as predictors of protective action following natural disaster. British Journal of Health Psychology, 2000; 5: 351-363.

57. Viscusi K. Smoking: Making the Risky Decision. New York: Oxford University Press, 1992.

58. Windschitl PD. Judging the accuracy of a likelihood judgment: The case of smoking risk. Journal of Behavioral Decision Making, 2002; 15: 19-35.

59. Peele S, Brodsky A. Exploring psychological benefits associated with moderate alcohol use: a necessary corrective to assessments of drinking outcomes? Drug and Alcohol 
Dependence, 2000; 60: 221-247.

60. Slovic P. Perceptions of risk. Science, 1987; 236: 280-285.

61. Hertwig R, Barron G, Weber EU, Erev I. Decisions from experience and the effect of rare events in risky choice. Psychological Science, 2004; 15: 534-539.

62. Garcia-Retamero R, Galesic M, Gigerenzer, G. Do Icon Arrays Help Reduce Denominator Neglect? Medical Decision Making, 2010; 30: 672-684.

63. Visschers VHM, Meertens RM, Passchier WWF, de Vries NNK. Probability information in risk communication: A review of the research literature. Risk Analysis, 2009; 29: 267-287.

64. Hampson SE, Andrews JA, Barckley M, Lee ME, Lichtenstein E. Assessing perceptions of synergistic health risk: A comparison of two scales. Risk Analysis, 2003; 23: 10211029.

65. Moravcsik JE, Kintsch W. Writing quality, reading skills, and domain knowledge as factors in text comprehension. Canadian Journal of Experimental Psychology, 1993; 47: $360-374$.

66. Kintsch W. Comprehension: A Paradigm for Cognition. New York: Cambridge University Press, 1998.

67. Hegarty M, Just MA. Constructing Mental Models of Machines from Text and Diagrams. Journal of Memory and Language, 1993; 32: 717-742. 


\section{TABLES AND FIGURES}

Table I. Participants' risk model judgments concerning the risk of developing esophageal cancer for individuals who are both heavy smokers and heavy drinkers. Judgments shown for each of the four risk communication conditions.

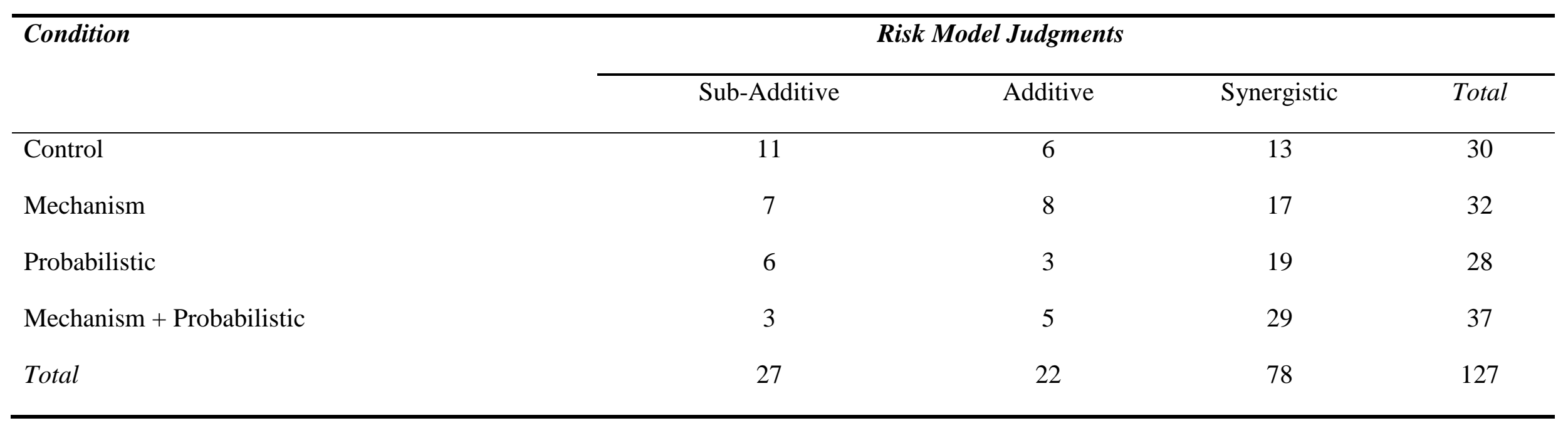


Table II. Mean ratings (standard deviations) in response to questions concerning the risk of developing esophageal cancer for individuals who are both regular smokers and regular drinkers.




Figure 1. Three simplified diagrams illustrating the hypothesized causal mechanism underlying the synergistic risk of esophageal cancer attributable to the combined use of alcohol and tobacco.

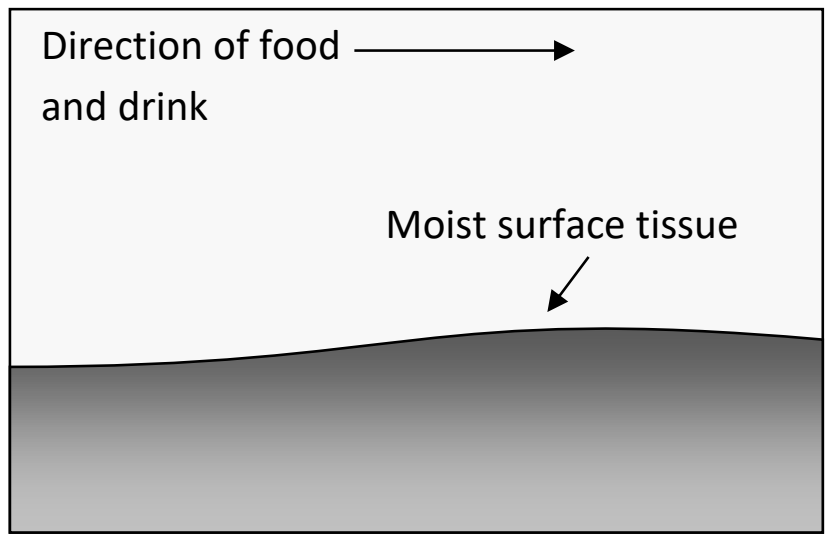

1. A cross-sectional view of the tissue lining of the esophagus.

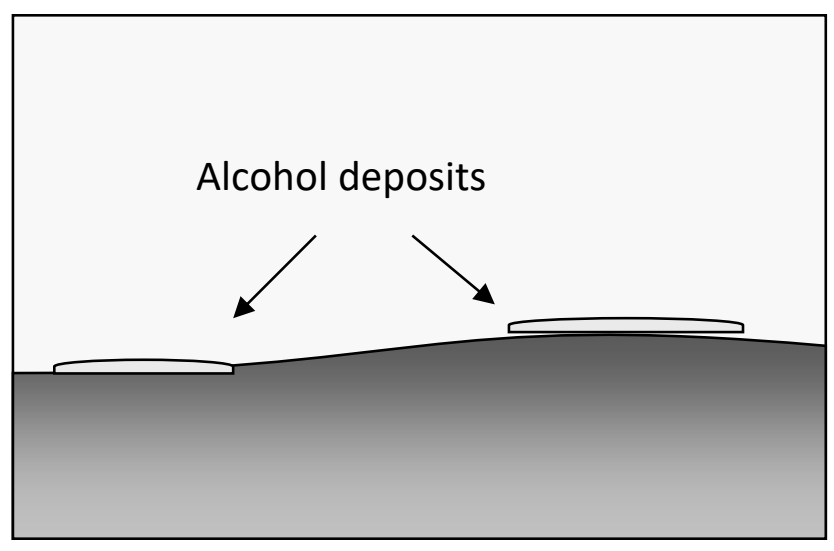

2. Small deposits of alcohol stick to the tissue lining of the esophagus.

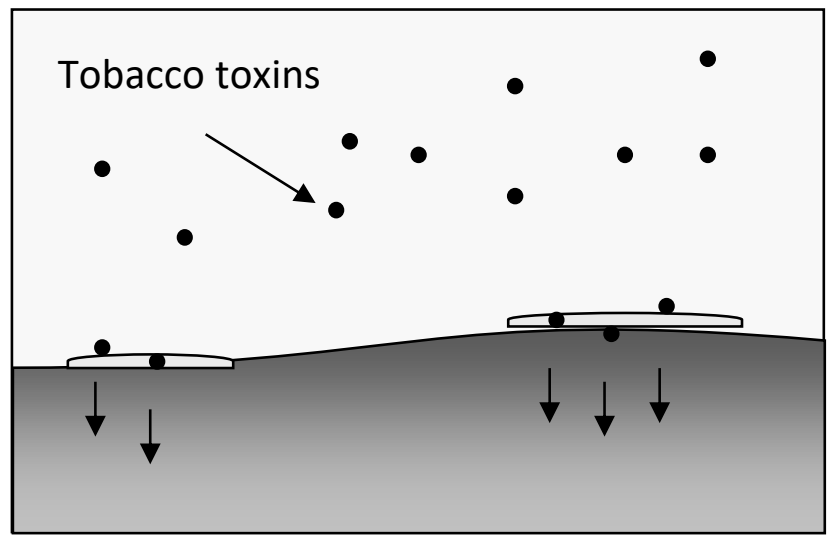

3. Tobacco toxins stick to the alcohol deposits and penetrate the tissue lining. 
Figure 2. Three icon arrays illustrating, respectively, the number of individuals who will develop esophageal cancer amongst a population of 100 heavy smokers, 100 heavy drinkers, and 100 individuals who both smoke and drink.

KEY: $\square=$ One person $\square=$ One person who will develop esophageal cancer

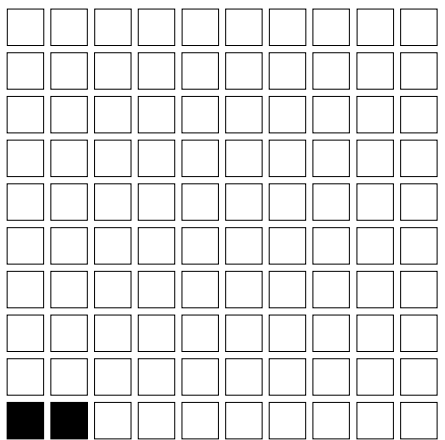

A population of 100 heavy smokers.

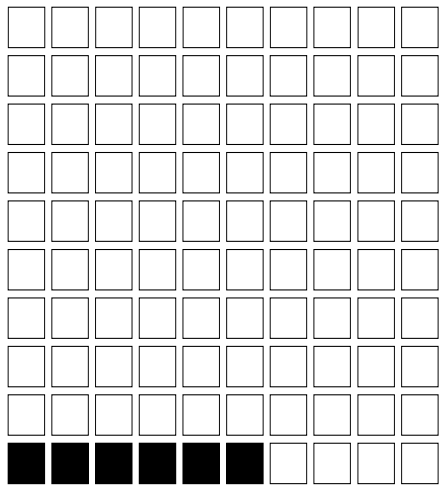

A population of 100 heavy drinkers.

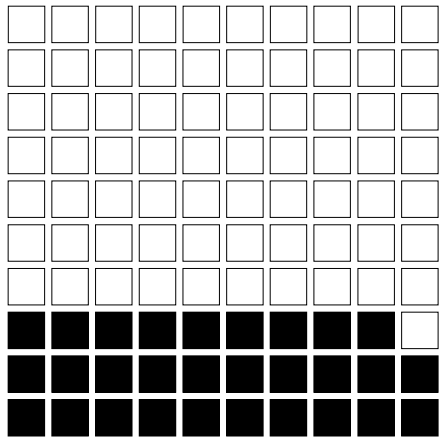

A population of 100 people; each one of whom is both a heavy smoker and a heavy drinker. 
Figure 3. Participants' risk model judgments concerning the risk of developing esophageal cancer for individuals who are both heavy smokers and heavy drinkers. Judgments shown for each of the four risk communication conditions and with the participants' sub-additive and additive risk model judgments collapsed into one category labeled as 'Non-Synergistic'.

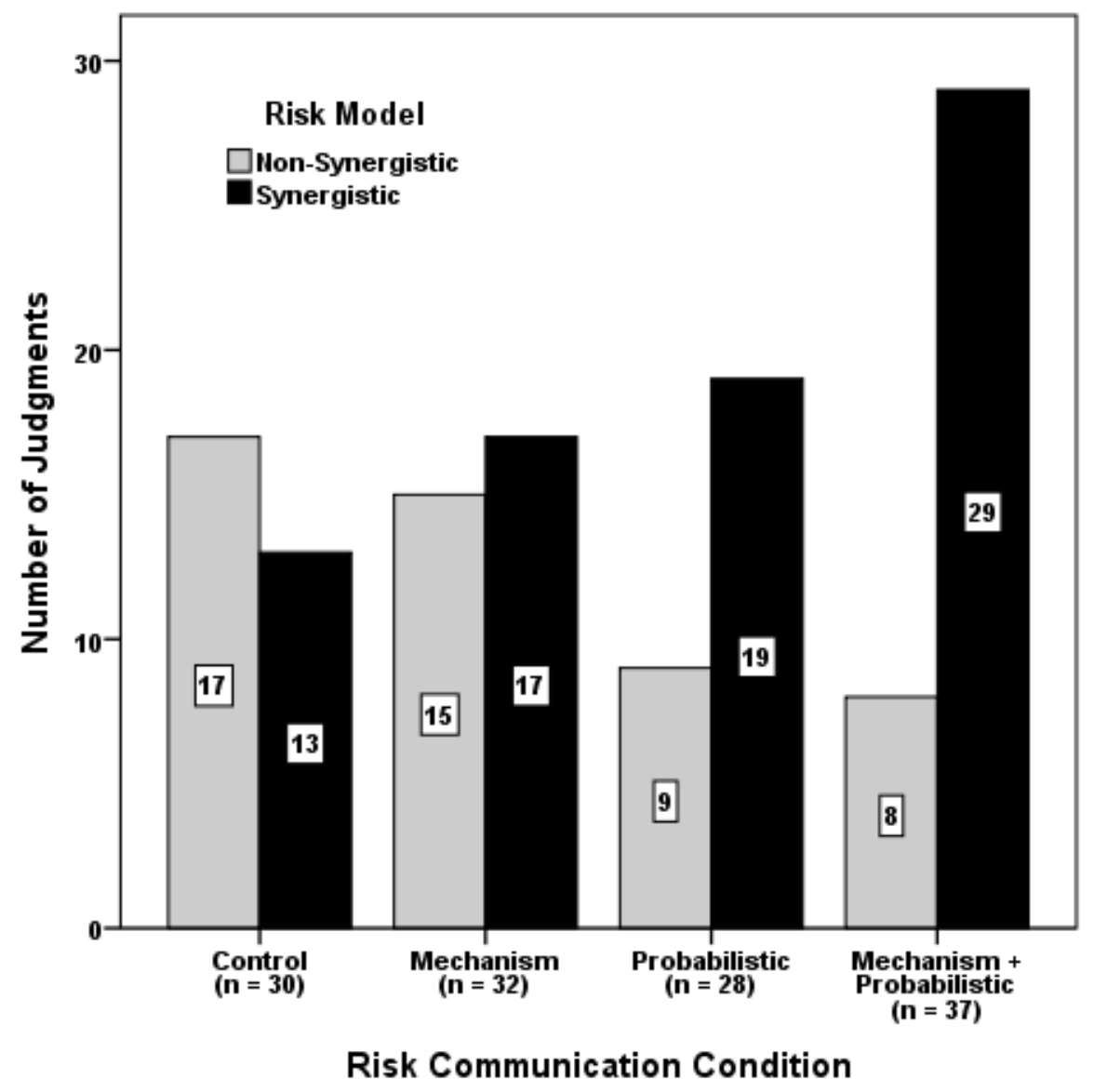


Figure 4. Participants' frequency estimates concerning the likelihood of developing esophageal cancer for individuals who are both heavy smokers and heavy drinkers. Estimates displayed in dichotomous categories of accurate $(+/-2$ of the 29/100 likelihood reported by Zambon et al., 2000) vs. inaccurate, and shown for each of the four risk communication conditions.

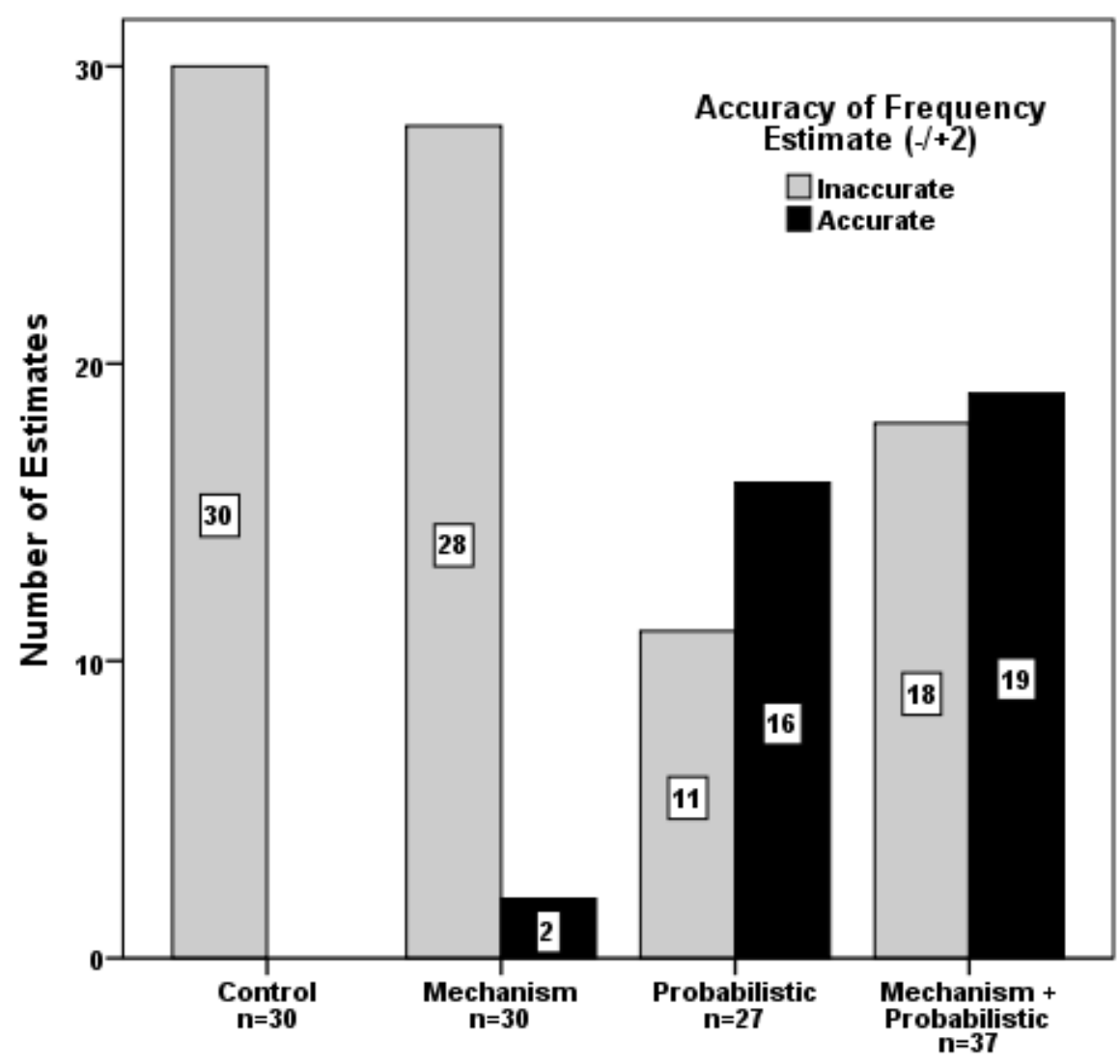

Risk Communication Condition 


\section{FOOTNOTES}

1. To address concerns that dichotomous splits can be associated with problems, such as loss of power, researchers have recently adopted an analytical approach that maintains numeracy as a continuous variable. ${ }^{(50,51)}$ Using the SPSS macro "PROCESS", we conducted a moderation analysis with numeracy (the moderator) as a continuous mean-centered variable, risk communication as the independent variable, and risk model as the binary (i.e., synergistic vs. non-synergsitic) dependent variable. ${ }^{(52)}$ Consistent with our hierachical loglinear analysis, the moderation analysis revealed no significant main or interaction effects for numeracy ( $c$ s < $-0.13, p s>0.27$ ). Furthermore, we repeated the 3-way loglinear analysis (risk model $\mathrm{x}$ risk communication $\mathrm{x}$ numeracy) using a trichotomous split for numeracy: low numeracy (score of 6 or less; $n=39$ ), medium numeracy (score of 7 or $8 ; n=56$ ) and high numeracy (score of

$9 ; \mathrm{n}=32)$. Again, there was no significant moderating effect for numeracy $\left(\chi^{2} \mathrm{~s}(6)<4.25, p \mathrm{~s}\right.$ > 0.64). Moreover, using "PROCESS", we also conducted a moderation analysis with numeracy (the moderator) as a continuous mean-centered variable, risk communication as the independent variable, and frequency estimate as the continuous dependent variable. Consistent with the hierachical loglinear analysis, the moderation analysis revealed no significant main or interaction effects for numeracy $(c \mathrm{~s}<0.12, p \mathrm{~s}>0.47)$. We also repeated the 3-way loglinear analysis (accuracy $\mathrm{x}$ risk communication $\mathrm{x}$ numeracy) using $\mathrm{a}$ trichotomous split for numeracy: low numeracy (score of 6 or less; $n=37$ ), medium numeracy (score of 7 or $8 ; n=56$ ) and high numeracy (score of 9; $\mathrm{n}=31$ ). Again, there was no significant moderating effect for numeracy $\left(\chi^{2} \mathrm{~s}(6)<4.62, p s>0.59\right)$. 
2. We recognized that a $+/-2$ criteria for an 'accurate' estimate provided participants with only a very small margin for error. We, therefore, repeated our three-way loglinear analysis (accuracy x risk communication x numeracy) but deemed estimates from 24 to 34 (i.e., +/-5) to be accurate and all other estimates deemed inaccurate. Consistent with the analysis using the $+/-2$ criteria, this identified a significant main effect for accuracy, $\chi^{2}(1)=9.44, p<0.01$. The main effect for accuracy was attributable to the greater proportion of participants, across the whole sample, who provided inaccurate (64\%) rather than accurate (36\%) frequency estimates, $\chi^{2}(1)=9.32, p<0.01$. Again, the main effect for accuracy was qualified by a twoway interaction between accuracy and risk communication, $\chi^{2}(3)=43.66, p<0.001$. Consistent with the original analyses, chi-square analyses identified that frequency estimates in both the control condition and the mechanism condition were predominantly inaccurate (93\% and $90 \%$ inaccurate, respectively), $\chi^{2} \mathrm{~s}(1)>19.19, p \mathrm{~s}<0.001$, and the frequency estimates in both the probabilistic condition and the mechanism+probabilistic were predominantly accurate (37\% and $38 \%$ inaccurate, respectively); again, the difference between the proportion of accurate/inaccurate estimates was not significant in either of these latter two conditions, $\chi^{2} \mathrm{~s}(1)<2.19, p \mathrm{~s}>0.05$. 